\title{
Zur Keratomykose beim Pferd
}

\author{
J. O. Bolliger, Margret B. Ruehli und B. M. Spiess \\ Veterinär-Chirurgische Klinik, Universität Zürich
}

\begin{abstract}
Zusammenfassung
Die Keratomykose beim Pferd ist eine äußerst gravierende und schwierig zu behandelnde Hornhauterkrankung, die nicht selten zum Verlust des Auges führt. Bei jeder stromalen Infiltration nach Hornhautverletzung und lokaler Antibiotika- und/oder Kortisonbehandlung soll eine Pilzkeratitis in Betracht gezogen und ein Hornhautzytologie vorgenommen werden. Absterbende Pilze setzen Toxine frei und verursachen eine massive Uveitis. Daher muss neben der lokalen antimykotischen, auch eine intensive lokale und systemische antiphlogistische Behandlung erfolgen. Um die Pilzbeladung der Kornea zu minimieren, empfiehlt es sich, zunächst ein Debridement oder eine Keratektomie des Infektionsherdes durchzuführen. Sitzen die Pilze tief stromal und besteht eine Perforationsgefahr, muss zusätzlich ein Bindehautlappen in den Hornhautdefekt eingenäht werden.

Am Beispiel von 18 Pferden, die in der Zeit von 1991 bis 1999 in unserer Klinik mit einer Keratomykose vorgestellt wurden, werden Symptomatik, Diagnostik sowie Therapiemöglichkeiten aufgezeigt.
\end{abstract}

Schlüsselwörter: Keratomykose, Pferd, Pilz, Diagnostik, Therapie,

\section{Equine Keratomycosis}

Equine keratomycosis is a severe disease, difficult to manage and always carrying a grave prognosis. Eyes may be lost due to fungal keratitis. Keratomycosis must be ruled out in every case of stromal keratitis, especially after previous glucocorticoid therapy. Corneal cytology is best suited to identify fungal elements. Degenerating fungi release endotoxins which cause a dramatic exacerbation of the accompanying uveitis. It is therefore mandatory to combine antifungal therapy with topical and systemic nonsteroidal antiinflammatory drugs. To decrease the load of fungi vigorous debridement and/or keratectomy is indicated. Deep stromal mycosis often necessitates conjunctival pedicle grafts or penetrating keratoplasties. The relevant literature is reviewed. Based on 18 cases seen between 1991 and 1999 in our clinic clinical signs, diagnosis and management of equine keratomycosis are discussed.

Keywords: $\quad$ keratomycosis, horse, fungi, diagnosis, therapy

\section{Einleitung und Literaturübersicht}

Die Keratomykose beim Pferd ist eine äußerst gravierende Hornhauterkrankung. Sie tritt in unseren Breitengraden meistens nach einem Korneatrauma und längerdavernden lokalen Antibiotika- und/oder Kortisonbehandlung am Auge auf. Da das Pferd in einer stark mit Pilzen belasteten Umwelt lebt ist der Infektionsdruck, im Gegensatz zum Kleintier, vergleichsweise hoch. Warm-feuchtes Klima (Ställe/südliche Gebiete) wirkt zudem begünstigend.

Pilzhyphen (Aspergillen, Fusarien, Penicillium, Alternaria, Cladosporium) wurden in der normalen Bindehautflora von Pferde isoliert (Davidson 1991). Am unverletzten Auge kann sich aber keine Keratomykose entwickeln. Damit es zu einer Pilzinfektion der Kornea kommt, braucht es eine vorausgehende Hornhautverletzung und weitere prädisponierende Faktoren. Dabei scheinen vor allem Kortikosteroide die natürliche, lokale Immunität zu beeinträchtigen und die Toleranzgrenze, das heißt Grenzdosis von okulierten Blastosporen, die der Organismus ohne äussere Krankheitszeichen vernichten kann, herabzusetzen. Über Antibiotika, als weiteren Risikofaktor, liegen unterschiedliche Ergebnisse vor. So soll Tetrazyklin unter $0.01 \%$ einen wachstumsfördernden, und bei $0.25 \%$ einen hemmenden Einfluß auf Candida albicans haben. Demgegenüber setzen in vitro Doxycylin, Neomycin und Gentamycin die minimale Hemmkonzen- tration von Candida sp. gegenüber Imidazol-Derivaten herab (Behrens-Baumann 1991). Es wird vermutet, daß der positive Einfluß der Antibiotika auf das Pilzwachstum durch direkte Wachstumsstimulation bewirkt wird. Auch soll durch die Eliminierung der Bakterien mit Verschiebung des bakteriellen Gleichgewichts eine "biologische Lücke" entstehen, in die Pilze hinein wachsen können (Behrens-Baumann 1991). Auch in anderen Experimenten mit Candida konnte, mit Ausnahme von Tetracyclin- $\mathrm{HCl}$, kein Einfluß von Antibiotika auf das Pilzwachstum festgestellt werden (Plempel 1984).

Die lokale, antibakterielle Prophylaxe wird teilweise empfohlen, weil das devitalisierte und nekrotische Stroma für bakterielle Infektionen prädisponiert ist (Davidson 1991).

In der Humanmedizin sind pflanzliche Fremdkörper Verursacher von Keratomykosen. Dabei können die Erreger direkt mit dem Fremdkörper in die Kornea gelangen oder bahnen durch die Hornhautverletzung der vorhandenen transienten Pilzflora den Weg.

Bei equinen Keratomykosen wurden am häufigsten Aspergillus spp., Fusarium spp., Penicillium spp. und Phycomyceten isoliert. Der beim Menschen häufig vorkommende Candida albicans wurde beim Pferd nur selten als Erreger einer mykotischen Keratitis gefunden (Davidson 1991). 
Eine frühzeitige Diagnose und intensive Behandlung sind von äußerster Wichtigkeit. Dennoch kommt es nicht selten aus folgenden Gründen zum Verlust des Auges (Brooks 1999):

- Die Pilze, die eine Affinität zur Descemet'schen Membran haben, wachsen rasch in die Tiefe und führen zur Korneaperforation und Panophthalmie.

- Absterbende Pilze setzen Toxine frei und führen zu einer massiven Uveitis und letztlich zur Phthisis bulbi.

In dieser Arbeit sollen Symptomatik, Diagnostik, sowie Therapie und Verlauf der Keratomykose aufgezeigt werden. Aufgrund der 18 vorgestellten Fälle, die zwischen 1991 und 1999 mit einer Keratomykose an der Veterinär-Chirurgischen Klinik der Universität Zürich untersucht wurden, werden Therapievorschläge gemacht.

\section{Symptomatik}

Betroffene Pferde haben meist eine längere Vorgeschichte mit chronischer Hornhautveränderung, welche nicht oder nur teilweise auf die Behandlung mit Kortikosteroiden und/oder Antibiotika anspricht. Die häufigsten Symptome sind:

\section{Blepharospasmus}

Je nach Tiefe und Ausdehnung der Infektion in der Hornhaut und abhängig davon, ob eine Uveitis vorliegt, zeigen betroffene Tiere mehr oder weniger starke Schmerzen.

\section{Hornhautveränderungen}

Das Hornhautepithel kann intakt sein, meist ist aber ein Ulkus erkennbar. Dessen Ränder sind nekrotisch und unscharf begrenzt. Das Stroma ist fokal oberflächlich oder tief gelblich infiltriert und zeigt mehr oder weniger starke Neovaskularisation (Abb. 1). Teilweise ist auch eine typische plaqueartige Auflagerung zu sehen (Abb. 2). Gelegentlich ist die Bildung von Satellitenherden zu beobachten.

Der Krankheitsprozess verläuft bei Keratomykosen langsamer als bei bakteriellen Infekten. Tief stromale Infiltrationen oder Abszessbildungen sprechen eher für eine Pilzinfektion, während oberflächlichere Herde meistens eher bakteriell bedingt sind.

\section{Uveitis anterior}

Vor Behandlungsbeginn ist häufig nur eine milde Begleitiritis mit minimaler Miosis und wenig Tränenfluss zu sehen, die sich unter der Behandlung, infolge von Toxinbildung, meist zu einer massiven Uveitis verstärkt.

\section{Diagnostik}

Vorgeschichte und klinisches Bild wecken oft Verdacht auf eine Keratomykose. Der Nachweis von Pilzen ist dann von ausschlaggebender Bedeutung.

Zu diesem Zweck muss Material für eine zytologische Untersuchung und für die Pilzkultur gewonnen werden.

Für die Entnahme von Material zur Zytologie, hat sich die aus der Humangynäkologie stammende Cytobrush' bewährt (Baver 1996) (Abb. 3). Die Ausstriche weisen eine hohe Zellzahl und exzellente
Zellverteilung auf, und zeigen weniger Zellschädigungen, als mit einem Spatel entnommene Proben. Die luftgetrockneten Ausstriche werden anschließend mit Giemsa, Lactophenolblau oder never Methylenblaulösung gefärbt (Baver 1999). Bei oberflächlichen Infiltraten, unter intaktem Korneaepithel, muss vor der Probeentnahme das Epithel durch Skarifikation entfernt werden.

Zur Diagnose von tief stromalen Infiltrationsherden ist oft eine Hornhautbiopsie nötig.

Mittels Zytologie können in den meisten Fällen Pilzhyphen rasch und zuverlässig nachgewiesen und so die Diagnose gesichert werden (Abb. 4).

Dabei liegen im Falle einer Keratomykose eine Vielzahl von Pilzhyphen umgeben von neutrophilen Granulozyten vor. Vereinzelte Pilzelemente ohne direkten Bezug zu entzündlichen Infiltraten sind als zufällige Kontamination zu betrachten, da Pilze zur physiologischen Bindehautflora des Pferdes gehören (Samuelson, Andresen et al. 1984).

Das Fehlen von Pilzen im zytologischen Präparat bedeutet allerdings nicht, daß eine Keratomykose ganz ausgeschlossen werden kann. Weil Pilzhyphen oft in der Tiefe des Stromas lokalisiert sind, muss eine Probe ausreichend tief entnommen werden, was in Einzelfällen schwierig und riskant sein kann.

Proben für eine Kultur sollten möglichst vom Rand der Läsion entnommen werden, da im Zentrum häufig nur sekundäre Mischflora angetroffen wird (Behrens-Baumann 1991). Viele Pilze können in 3-4 Tagen angezüchtet werden, während es bei anderen hingegen Wochen dauern kann (Davidson 1991).

Korneabiopsien sollten für das Anlegen einer Kultur und zur histologischen Untersuchung verwendet werden. Eine Typisierung des vorliegenden Pilzes kann bei der Auswahl der Medikamente hilfreich sein (Coad, Robinson et al. 1985; Brooks, Andrew et al. 1998).

\section{Therapie}

\section{Medikamentelle antimykotische Behandlung}

Da eine systemische Behandlung von Keratomykosen beim Pferd aus finanziellen Gründen meist nicht in Betracht kommt, und zudem relativ geringe Gewebekonzentrationen in der Hornhaut erzielt werden, steht die lokale antimykotische Behandlung im Vordergrund (O'Day 1990).

Die Antimykotika können in 4 Gruppen unterteilt werden (Behrens-Baumann 1997):

- Polyene (Natamycin, Amphotericin, Nystatin)

- Pyrimidine (5-Fluorcytosin)

- Imidazolderivate (Miconazol, Fluconazol, Itraconazol, usw. )

- andere (Silber-Sulfadiazine, Povidone-lodine)

\section{Polyene}

\section{Natamycin}

Natamycin wird als Mittel der Wahl zur Behandlung von Keratomykosen beim Pferd angesehen. Es hat das breiteste Spektrum gegen pathogene Pilze (Davidson 1991). Wegen seiner 
schlechten Löslichkeit ist seine Penetration limitiert (BehrensBaumann 1991; Behrens-Baumann 1997). Daher wird es vor allem bei oberflächlichen Keratomykosen eingesetzt. Es wirkt bei lokaler Applikation am Auge leicht irritierend. Subkonjunktivale Injektionen können zu Nekrosen führen und sollten vermieden werden. In vitro besteht eine gute Wirksamkeit gegenüber Candida, Aspergillus, Penicillium und Fusarium. Insbesondere gegen Fusarium Sp. ist die Aktivität deutlich besser, als die der Imidazol-Derivate (Behrens-Baumann 1991). Natamycin ist als einziger Wirkstoff als ophthalmologisches Präparat ${ }^{2,3}$ erhältlich. Der Nachteil von Natamycin ist sein hoher Preis.

\section{Amphotericin B}

Amphotericin $B^{4}$ wirkt lokal irritierend und verzögert die korneale Epithelisierung (Behrens-Baumann 1997). Es wird als 1-10\%ige Lösung (verdünnt mit Aqua ad inj. oder 5\%iger Dextrose) lokal alle 2-4 Stunden appliziert. Die Korneapenetration ist schlecht. Sein Vorteil liegt darin, daß auch die subkonjunktivale Injektion von $125 \mu \mathrm{g}$ Amphotericin (3 x wöchentlich) möglich ist (Barnett 1995). Amphotericin B hat eine fungizide Wirkung gegen Aspergillus, Mucor, Fusarium, Alternaria und Candida (Regnier, Whitley et al. 1986). Es lassen sich allerdings auch Resistenzen von Candida sp. und Aspergillus sp. finden (Behrens-Baumann 1991).

\section{Pyrimidine}

\section{5-Fluorcytosin}

Fluorcytosin kann als 1\%ige Lösung auf das Auge getropft werden. Es ist nicht irritierend und besitzt keine toxische Wirkung (Behrens-Baumann 1991; Davidson 1991). Es zeichnet sich durch eine gute Korneapenetration aus und wurde erfolgreich bei CandidaKeratitiden eingesetzt (Behrens-Baumann 1991). Es muss jedoch bei alleiniger Anwendung unter der Behandlung mit der Entwicklung von Resistenzen gerechnet werden.

\section{Imidazolderivate}

\section{Miconazol}

Die $1 \%$ ige Lösung von Miconazol ${ }^{5}$ kann direkt auf das Auge getropft und auch subkonjunktival (5-10 mg alle 24 bis 48 Stunden) injiziert werden. Es besitzt eine gute korneale Penetration und scheint nicht zu irritieren. Zusammen mit Fluorcytosin behindert es von allen Antimykotika am wenigsten die Reepithelisierung der Kornea. Es hat den Vorteil, daß es neben der fungiziden auch eine antibakterielle Wirkung aufweist (BehrensBaumann 1991; Brooks 1999). Miconazol wurde auch mit Erfolg intrakameral injiziert, in der Dosierung von $100 \mu \mathrm{g} /$ Auge (Pickett 1995). Sein Wirkungsspektrum beinhaltet Aspergillus, Penicillium, Alternaria, Fusarium, Curvularia und Pseudoallescheria. Fusariumspezies können allerdings resistent sein (Behrens-Baumann 1991; Behrens-Baumann 1997).

In einer Studie wurde bei 35 von 39 Patienten Miconazol als alleinige medikamentelle Therapie eingesetzt (Andrew 1998). Bei 20 Pferdeaugen konnte so das Sehvermögen erhalten werden. Bei weiteren 19 Patienten, bei denen eine kombinierte medikamentelle und chirurgische Therapie durchgeführt wur- de, verloren 3 Patienten das Sehvermögen.

Fluconazol

Als Alternative zu Miconazol kann auch Fluconazol6 verwendet werden (Urbak and Degn 1994). Es hat ein ähnliches Wirkspektrum wie Miconazol (Thakar 1994).

\section{Itraconazol}

Neuerdings wird auch die lokale Applikation von Itraconazol7 in DMSO propagiert (Negroni and Arechavala 1993; Ball 1997). Die Autoren haben damit aber noch keine eigenen Erfahrungen.

Andere

\section{Silber-Sulfadiazine}

Silber-Sulfadiazine hat antibakterielle und fungizide Wirkung. Es penetriert gut in die Hornhaut. Silbernitrat denaturiert Proteine und zerstört auch Epithelzellen. Es wirkt leicht irritierend und wird als $1 \%$ ige Hautsalbe ${ }^{8}$ direkt auf das Auge verabreicht (Bron, Cellier et al. 1990). Sein Wirkungsspektrum beinhaltet Aspergillus, Fusarium und Fadenpilze. Bei einer Studie wurden 1\%ige Silber-Sulfadiazin-Tropfen mit 1\%igen Miconazol-Tropfen verglichen. Dabei zeigte sich die Wirkung der Silber-SulfadiazinTropfen gegenüber den Miconazol-Tropfen deutlich überlegen (Bron, Cellier et al. 1990; Vajpayee, Gupta et al. 1990).

\section{Povidone-lodine-Lösung}

Die direkte Spülung mit Povidone-lodine-Lösung ${ }^{9}(1: 10$ bis 1:50 mit $\mathrm{NaCL}$ verdünnt) wurde ebenfalls zur Behandlung von Keratomykosen empfohlen (McLaughlin, Brightman et al. 1983; Davidson 1991). Es besitzt fungizide und bakterizide Wirkung.

\section{Chirurgische Therapie}

Das Korneaepithel scheint die wesentliche Barriere für die Bioverfügbarkeit von Antimykotika im Hornhautstroma darzustellen. Eine Abrasio corneae erhöht die Konzentration der Antimykotika erheblich (Behrens-Baumann 1997).

In der Humanmedizin wird die Keratektomie vor allem aus diagnostischen Gründen, zur Verbesserung der Pharmakokinetik und zur partiellen Entfernung von Erreger, als Unterstützung der medikamentellen Behandlung eingesetz† (Behrens-Baumann 1997). Bei tief liegenden Prozessen wird aber die perforierende Keratoplastik angewendet. Bei einer Studie an Kaninchenaugen, die mit Aspergillus fumigatus infiziert wurden, gingen alle Augen, die mit lamellärer Keratoplastik behandelt wurden, verloren, während von 10 Augen mit perforierender Keratoplastik 8 erhalten werden konnten (Behrens-Baumann 1991).

Bei der Behandlung von Pferden mit Keratomykosen erscheint neben der medikamentellen eine chirurgische Behandlung zur Reduktion der Erregerdichte sinnvoll (Brooks 1999). Liegen demarkierte Hornhautbezirke vor, so können diese am stehenden Pferd in Sedation und Lokalanästhesie entfernt werden. Eine großräumige Dekontamination der infiltrierten Kornea kann mittels einer Keratektomie am liegenden Pferd erfolgen. Anschließend muss ein gestielter Bindehautlappen eingenäht werden (Brooks 1999) (Abb. 5). Ein Bindehautlappen bringt Blutgefäße an den Ort des Geschehens, so daß besonders auch subkonjunktival verabreichte Medikamente effizient einwirken können. Der frei- 
liegende Stiel des Bindehautlappens kann nach einigen Wochen durchtrennt und entfernt werden (Abb. 6). Die einge-

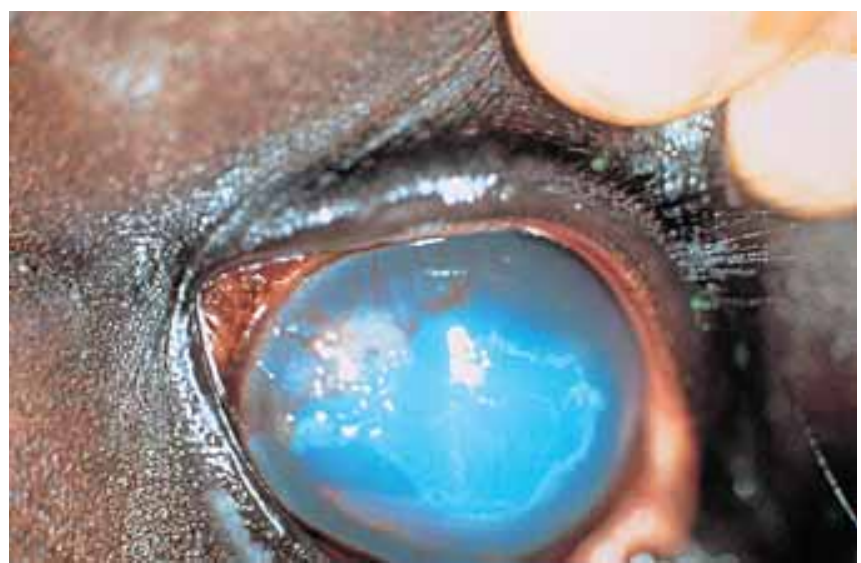

Abb. 1: Stromale Infiltration bei einem Pferd mit Keratomykose (Fall Nr. 2).

Stromal infiltrate in a horse with kertomycosis (Case Nr. 2).

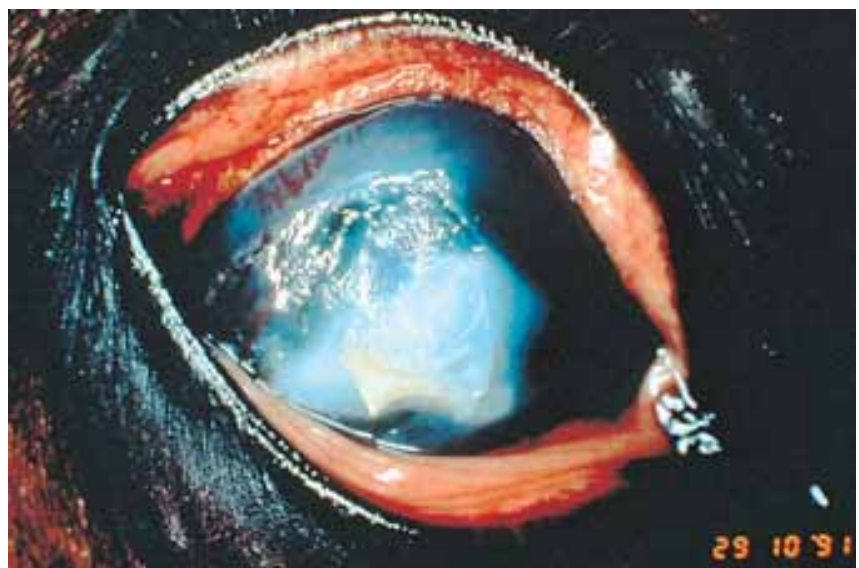

Abb. 2: Plaqueartige Hornhautauflagerungen bei einem Pferd mit Keratomykose (Fall Nr. 7)

Plaque-like corneal deposit in a horse with keratomycosis (Case Nr. 7).

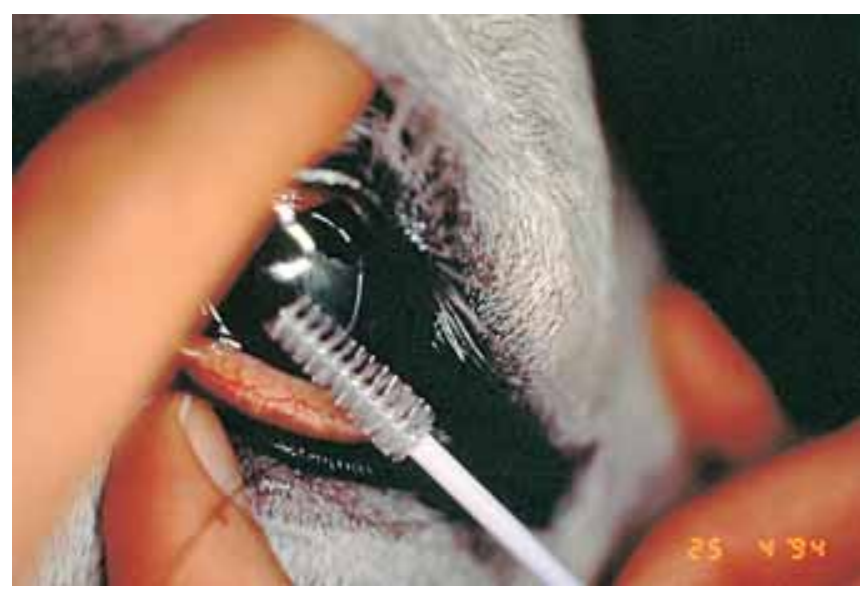

Abb. 3: Entnahme einer Korneazyłologie mittels eines CytoBrush.

Exfoliative corneal cytology using a Cytobrush.

wachsene Bindehaut führt zu einer dichten Narbe (Abb. 7) Antibiotische Behandlung

Der Einsatz von Antibiotika in Fällen von Keratomykose ist sehr kontrovers (Behrens-Baumann 1991; Behrens-Baumann 1997; Brooks 1999). Wo einige Autoren Gentamicin und andere Antibiotika lokal verabreichen raten andere strikt davon ab. Hier müssen von Fall zu Fall die Anamnese, das klinische Bild, sowie die zytologischen und bakteriellen Resultate mit in

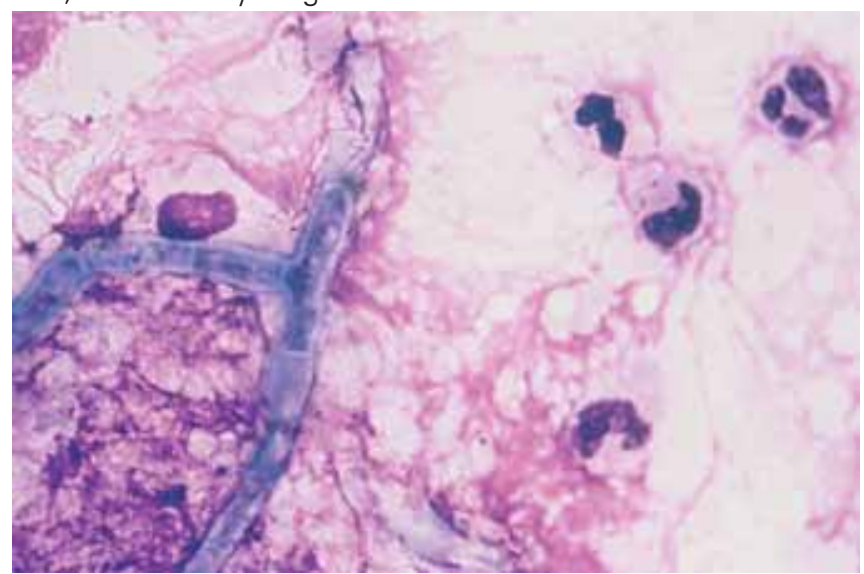

Abb. 4: Hornhautzytologie eines Pferdes mit Keratomykose: Septiertes und verzweigtes Pilzelement (Aspergillus) und neutrophile Granulozyten.

Corneal cytology of a horse with keratomycosis: Septated and branching fungal elements (Aspergillus) and neutrophils.

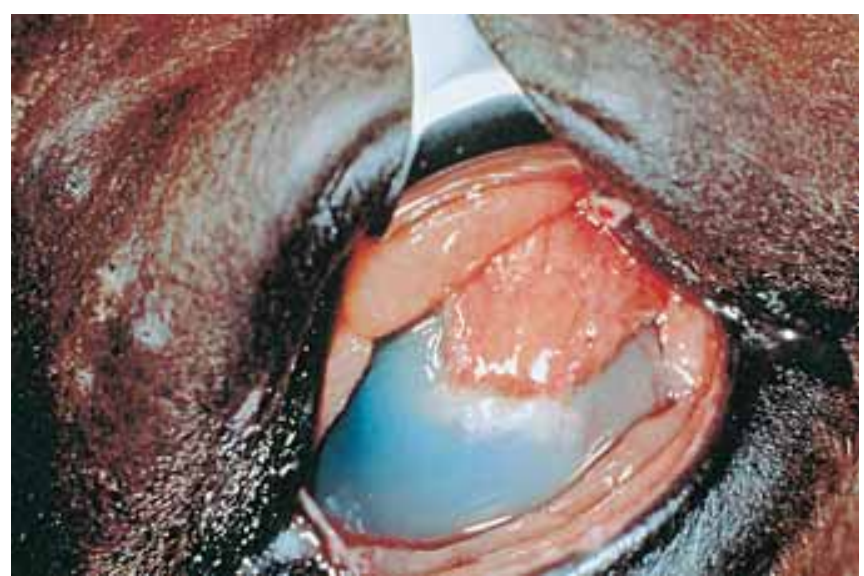

Abb. 5: Bindehautlappen nach Keratektomie (Fall Nr. 12).

Conjunctival pedicle flap after keratetomy in case Nr. 1.2.

den Entscheid einbezogen werden.

Behandlung der Begleitiritis

In jedem Fall muss gleichzeitig zur antimykotischen Therapie auch eine antiphlogistische Behandlung erfolgen. Kortikosteroide sind immer zu vermeiden (Brooks 1999). Lokale nicht-steroidale Entzündungshemmer, wie z.B. Diclofenac- $\mathrm{Na}^{10}$, müssen mit systemischen kombiniert werden. Gut geeignet sind dafür Flunixin Meglumine 11 oder Vedaprofen ${ }^{12}$

Daneben ist die Zykoplegie sehr wichtig. Atropin ${ }^{13}$ kann zum Weitstellen der Pupille bis dreimal täglich verabreicht werden. Genügt das nicht, muss eine subkonjunktivale Sprengspritze ${ }^{14}$ verabreicht werden.

\section{Adjuvante Therapie}

Um die intensive Behandlung des Auges sicherzustellen, empfiehlt es sich, einen Behandlungsschlauch ${ }^{15}$ durch das Oberlid 
einzulegen (Spiess 1994) (Abb. 8). Das Anbringen von Augenverbänden oder -kappen soll unterbleiben, weil sich darunter ein feuchtwarmes Klima entwickelt, welches das Pilzwachs-

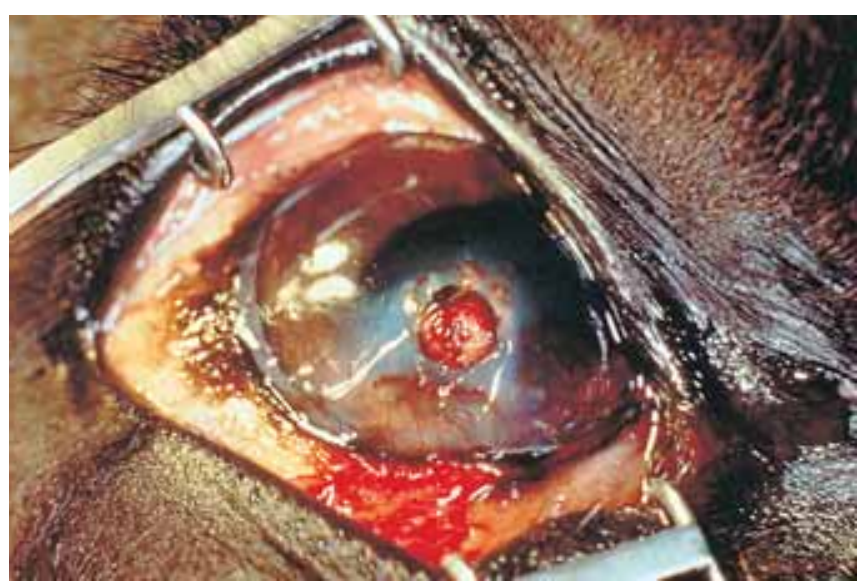

Abb. 6: Bindehautlappen unmittelbar nach Durchtrennen des freiliegenden Stiels (Fall Nr. 13)

Conjunctival pedicle flap immediately after transsection of the unattached.

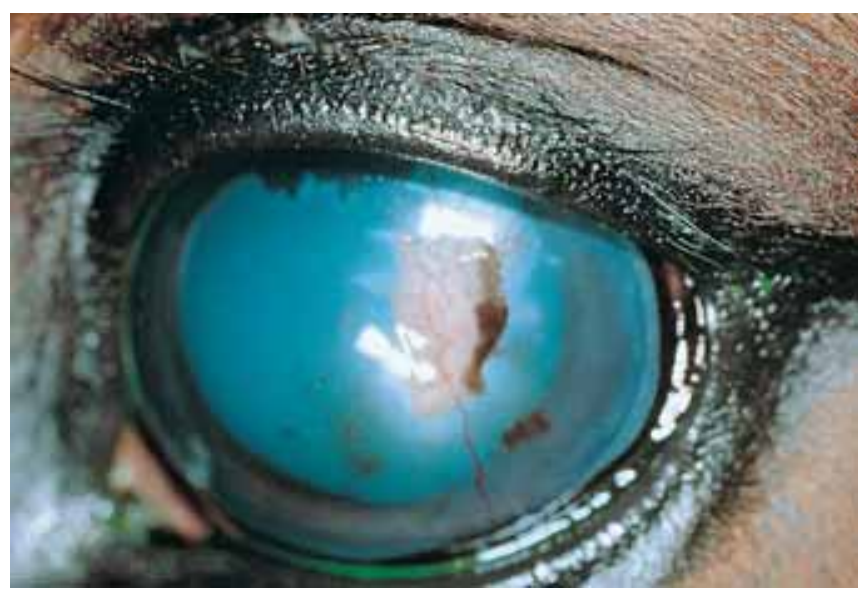

Abb. 7: Hornhautnarbe durch eingewachsenen Bindehautlappen (Fall Nr. 16): Zustand 3 Wochen nach Durchtrennen des Stiels.

Corneal scar after a conjunctival pedilce graft (Case Nr. 16): 3 weeks after transsection of the pedicle.

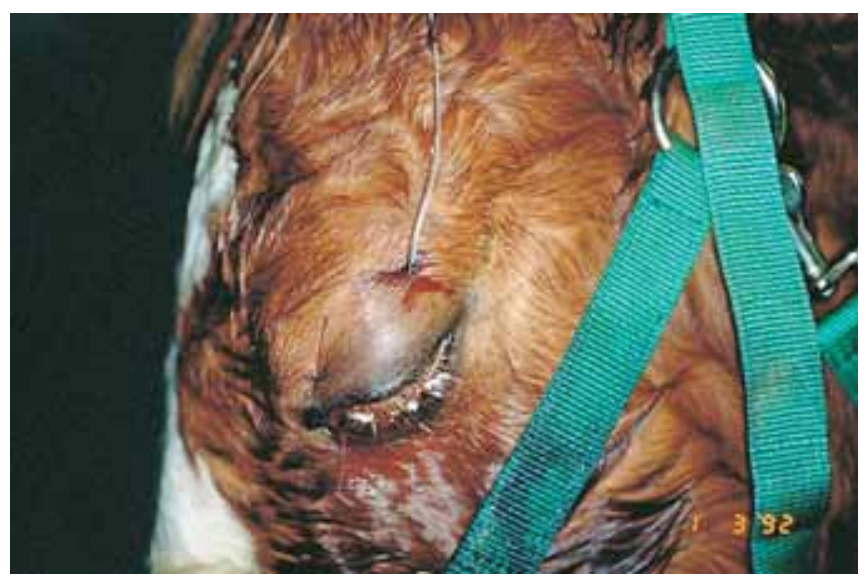

Abb. 8: Pferd mit einem Behandlungsschlauch durch das Oberlid.

Subpalpebral lavage system in the superior lid of a horse. tum begünstigt.

\section{Prognose}

Grundsätzlich muss die Prognose beim Vorliegen einer Keratomykose immer äußerst vorsichtig gestellt werden. Im besten Fall kommt es unter Bildung einer Hornhautnarbe im Bereich des Infektionsherdes zur vollständigen Abheilung und Erhaltung des Sehvermögens. Häufig treten Komplikationen auf, wie Hornhautperforation, septische Endophthalmitis oder unkontrollierbare Uveitis, die alle zum Verlust des Auges führen.

\section{Eigene Fälle}

In der Zeit von 1991 bis 1999 wurden 18 Pferde mit einer Keratomykose in unserer Klinik vorgestellt (Tab. 1). Zwölf der 18 Patienten wurden mit Kortikosteroiden vorbehandelt, fünf mit Antibiotika und eines mit einem Virustatikum. In 16 Fällen konnten Pilze zytologisch nachgewiesen werden. In einem Fall gelang der Nachweis nur mittels Biopsie (Nr. 1), und in einem weiteren Fall (Nr. 3) erst mittels histologischer Untersuchung des enukleierten Auges. In 7 Fällen konnten Aspergillus sp. auch in der Kultur identifiziert werden. Der häufigste Eintrittsbefund waren Hornhautulzerationen, welche teilweise nekrotisierend waren, teils durch weissliche, festhaftende Beläge charakterisiert waren. In zwei Fällen bestand ein tief stromaler Abszess. Eine mehr oder weniger ausgeprägte Begleitiritis war in allen Fällen diagnostiziert worden. In 6 Fällen kam es zum Verlust des Auges, in einem Fall zur Phthisis bulbi. Die übrigen 11 Fälle resultierten in einer Hornhautnarbe unterschiedlicher Größe und Dichte bei erhaltenem funktionellem Sehvermögen (Tab. 2). Neun Pferde wurden ausschließlich medikamentell behandelt. Dabei kam es in 4 Fällen zum Verlust des Auges, in einem Fall zur Phthisis bulbi. In 4 Fällen heilte die Hornhaut unter Narbenbildung ab. In 8 Fällen wurde zusätzlich zur medikamentellen Therapie eine Keratektomie mit gestieltem Bindehautlappen durchgeführt. Nur in einem Fall führte dies zum Verlust des Auges. Ein Fall wurde keratektomiert ohne Bindehautlappen, was ebenfalls die spätere Enukleation nach sich zog. Die Pferde wurden durchschnittlich während 19.5 Tagen hospitalisiert (7 bis 34 Tage).

\section{Diskussion}

Einer Keratomykose geht immer ein Hornhauttrauma voraus. In unserem Patientengut scheint auch eine Vorbehandlung mit Kortikosteroiden und/oder Antibiotika die Voraussetzung für eine Pilzinfektion zu sein.

Das klinische Bild reicht für eine Diagnose alleine nicht aus. Zwar sind weißliche, festhaftende Beläge recht typisch für Keratomykosen, doch werden sie nicht regelmäßig gesehen. Der Pilznachweis gelingt aber mittels Zytologie in der Mehrzahl der Fälle. Die Technik ist einfach und wenig invasiv. An unserer Klinik wird jedes Ulkus beim Pferd auch zytologisch untersucht. Der Vorteil der Zytologie ist, daß ein Resultat bereits nach kurzer Zeit, meist innerhalb einer Stunde, vorliegt. Werden Kulturen angelegt, vergehen mehrere Tage bis Wochen, bis ein Re- 
Tab. 1: Signalement, Vorbehandlung, Aufenthaltsdaver und initiale Befunde der 18 Pferde mit Keratomykose.

Signalment, previous treatment, duration of hospitalization and initial ophtalmic findings of 18 horses with keratomycosis.

\begin{tabular}{|c|c|c|c|c|}
\hline Fall $\mathrm{Nr}$. & Signalement & Vorbehandlung & $\begin{array}{l}\text { Spital- } \\
\text { tage }\end{array}$ & Initialer Befund \\
\hline 1 & Stute, Welsh Cob, $21 \mathrm{Jh}$. & AB, Atropin, FM, NHS & 14 Tage & Ulkus, Uveitis anterior \\
\hline 2 & Stute, Araber, $14 \mathrm{Jh}$. & $\begin{array}{l}\text { Lokale Kortikosteroide } \\
\text { über } 3 \text { Wochen }\end{array}$ & 30 Tage & Oberflächliches Ulkus \\
\hline 3 & Stute, Haflinger, $10 \mathrm{Jh}$. & $\begin{array}{l}\text { Atropin, } \\
\text { Trifluorothymidin }\end{array}$ & 27 Tage & $\begin{array}{l}\text { Korneaabszess mit endothelialen } \\
\text { Ablagerungen und Uveitis ant. }\end{array}$ \\
\hline 4 & Wallach, Holland, 6 Jh. & $\begin{array}{l}\text { Lokale Steroide über } 3 \\
\text { Wochen }\end{array}$ & 20 Tage & Kornealazeration mit losem Lappen \\
\hline 5 & Wallach, Westfahlen, $9 \mathrm{Jh}$. & $\begin{array}{l}\text { Atropin, Acetylcystein, } \\
A B\end{array}$ & 16 Tage & Ulkus mit zentraler Keratomalazie \\
\hline 6 & Wallach, Holland, 23 Jh. & $\begin{array}{l}\text { AB und Kortikosteroide } \\
\text { lokal } 2 \text { Wochen }\end{array}$ & 10 Tage & nekrotisierendes Ulkus \\
\hline 7 & Wallach, Holstein, $6 \mathrm{Jh}$. & $\begin{array}{l}\text { AB und lokale } \\
\text { Kortikosteroide }\end{array}$ & 11 Tage & $\begin{array}{l}\text { Oberflächliche Keratitis mit weissl. } \\
\text { Auflagerungen }\end{array}$ \\
\hline 8 & Stute, Schweiz, 8 Jh. & $\begin{array}{l}\text { Lokale Kortikosteroide } \\
\text { über } 3 \text { Wochen }\end{array}$ & 10 Tage & $\begin{array}{l}\text { Keratitis, endotheliale } \\
\text { Ablagerungen, Uveitis ant. }\end{array}$ \\
\hline 9 & Wallach, Schwarzwald, 9 Jh. & $\begin{array}{l}\text { AB systemisch, } \\
\text { Kortikosteroide lokal } \\
\text { und systemisch über } 8 \\
\text { Tage }\end{array}$ & 7 Tage & $\begin{array}{l}\text { Nekrotisierendes Ulkus und Uveitis } \\
\text { ant. }\end{array}$ \\
\hline 10 & Stute, Schweiz, $10 \mathrm{Jh}$. & $\begin{array}{l}\text { Lokale Kortikosteroide } \\
\text { über } 2 \text { Wochen }\end{array}$ & 28 Tage & Ulkus mit weissl. Bel\%ogen \\
\hline 11 & Wallach, Schweiz, $7 \mathrm{Jh}$. & $A B$ während 10 Tagen & 17 Tage & $\begin{array}{l}\text { Nekrotisierendes Ulkus und Uveitis } \\
\text { ant. }\end{array}$ \\
\hline 12 & Stute, Schweiz, $10 \mathrm{Jh}$. & $\begin{array}{l}\text { Lokale } A B \text { und } \\
\text { Kortikosteroide }\end{array}$ & 19 Tage & $\begin{array}{l}\text { Kornealazeration mit } \\
\text { nekrotisierendem Lappen }\end{array}$ \\
\hline 13 & Stute, Araber, $12 \mathrm{Jh}$. & $\begin{array}{l}\text { Lokale } A B \text { und } \\
\text { Kortikosteroide }\end{array}$ & 34 Tage & Nekrotisierendes Ulkus \\
\hline 14 & Wallach, Holland, $6 \mathrm{Jh}$. & $\begin{array}{l}\text { Lokale } A B \text { während } 2 \\
\text { Wochen }\end{array}$ & 17 Tage & Nekrotisierendes Ulkus \\
\hline 15 & Stute, Schweiz, 7 Jh. & $\begin{array}{l}\text { Lokale AB und Kortiko- } \\
\text { steroide über } 2 \text { Wochen }\end{array}$ & 21 Tage & Korneabaszess mit Uveitis ant. \\
\hline 16 & Stute, Westfahlen, $10 \mathrm{Jh}$. & $\begin{array}{l}\text { Lokale } A B \text { und } \\
\text { Kortikosteroide }\end{array}$ & 32 Tage & $\begin{array}{l}\text { Nekrotisierendes Ukkus und Uveitis } \\
\text { ant. }\end{array}$ \\
\hline 17 & Wallach, Island, $10 \mathrm{Jh}$. & $\begin{array}{l}\text { Lokale AB und Kortiko- } \\
\text { steroide über } 4 \text { Wochen }\end{array}$ & 21 Tage & $\begin{array}{l}\text { Nekrotisierendes Ulkus mit Uveits } \\
\text { ant. }\end{array}$ \\
\hline 18 & Wallach, Schweiz, $13 \mathrm{Jh}$. & Lokale AB & 18 Tage & Nekrotisierendes Ulkus \\
\hline
\end{tabular}

AB: Antibiotika FM: Flunixin meglumine NHS: Nickhautschürze 
Tab. 2: Resultate der Zytologie und Pilzkultur, Behandlung und Resultat.

Results of cytology, fungal cultures, treatment and final outcome.

\begin{tabular}{|c|c|c|c|c|c|}
\hline $\begin{array}{l}\text { Fall } \\
\text { Nr. }\end{array}$ & $\begin{array}{l}\text { Zytologie/ } \\
\text { Biopsie }\end{array}$ & Kultur & Medikamentelle Behandlung & $\begin{array}{l}\text { Chirurgische } \\
\text { Behandlung }\end{array}$ & Resultat \\
\hline 1 & B: Pilzhyphen & - & Amphotericin B, Atropin & Enukl. nach 11 Tagen & Verlust des Auges \\
\hline 2 & Z: Pilzyhyphen & Asperg. sp. & $\begin{array}{l}\text { Amphotericin B, Miconazol, Clotrimazol, } \\
\text { Gentamicin, Atropin, SS }\end{array}$ & - & $\begin{array}{l}\text { Korneanarbe, } \\
\text { Visus erhalten }\end{array}$ \\
\hline 3 & H: Pilzhyphen & - & $\begin{array}{l}\text { Gentamicin, Atropin, Dexamethason } \\
\text { nach } 11 \text { Tagen Miconazol }\end{array}$ & $\begin{array}{l}\text { Keratekt. nach } 11 \text { Tagen, } \\
\text { Enukl. nach } 18 \text { Tagen }\end{array}$ & Verlust des Auges \\
\hline 4 & Z: Pilzhypgen & - & $\begin{array}{l}\text { Amphotericin B, Silber-Sulfadiazin, } \\
\text { Atropin }\end{array}$ & - & $\begin{array}{l}\text { Korneanarbe, } \\
\text { Visus erhalten }\end{array}$ \\
\hline 5 & Z: Pilzyhphen & - & $\begin{array}{l}\text { Amphotericin B, Silber-Sulfadiazine, } \\
\text { Atropin, FM }\end{array}$ & - & $\begin{array}{l}\text { Korneanarbe, } \\
\text { Visus erhalten }\end{array}$ \\
\hline 6 & Z: Pilzhyphen & Asperg. sp. & $\begin{array}{l}\text { Amphotericin B, Silber Sulfadiazine, } \\
\text { Atropine, FM }\end{array}$ & Enukl. nach 2 Tagen & Verlust des Auges \\
\hline 7 & Z: Pilzhyphen & - & Silber-Sulfadiazine, Jodlösung, Atropin & Enukl. nach 7 Tagen & Verlust des Auges \\
\hline 8 & $\begin{array}{l}\text { Z: Pilzhyphen im } \\
\text { Kammerwasser }\end{array}$ & - & $\begin{array}{l}\text { Miconazol lokal, subconjunctival, intra- } \\
\text { cameral, Silber-Sulfadiazin, Atropin, FM }\end{array}$ & $\begin{array}{l}\text { Keratekt., BHL nach } 3 \\
\text { und Enukl. nach } 7 \text { Tagen }\end{array}$ & Verlust des Auges \\
\hline 9 & Z: Pilzhyphen & Asperg. sp. & $\begin{array}{l}\text { Miconazol lokal und subconj. Atropin, } \\
\text { FM }\end{array}$ & Enukl. nach 4 Tagen & Verlust des Auges \\
\hline 10 & Z: Pilzhyphen & Asperg. sp. & $\begin{array}{l}\text { Miconazol lokal und subconi., Silber } \\
\text { Sulfadiazin, Atropin, FM, Acetylcystein }\end{array}$ & - & Phthisis bulbi \\
\hline 11 & Z: Pilzhyphen & Asperg. sp. & $\begin{array}{l}\text { Miconazol lokal und subconj., Silber } \\
\text { Sulfadiazin, Atropin, FM, }\end{array}$ & Keratekt., BHL & $\begin{array}{l}\text { Korneanarbe, } \\
\text { Visus erhalten }\end{array}$ \\
\hline 12 & $\begin{array}{l}\text { Z: Pilzhyphen } \\
\text { B: Keratitis mit } \\
\text { massivem } \\
\text { Pilzbefall }\end{array}$ & - & $\begin{array}{l}\text { Miconazol lokal und subconi., Silber } \\
\text { Sulfadiazin, Atropin, FM, }\end{array}$ & Keratekt., BHL & $\begin{array}{l}\text { Korneanarbe, } \\
\text { Visus erhalten }\end{array}$ \\
\hline 13 & Z: Pilzhyphen & - & $\begin{array}{l}\text { Amphotericin B, Miconazol lokal und } \\
\text { subconi., Atropin, FM, }\end{array}$ & Keratekt., BHL & $\begin{array}{l}\text { Korneanarbe, } \\
\text { Visus erhalten }\end{array}$ \\
\hline 14 & Z: Pilzhyphen & - & $\begin{array}{l}\text { Miconazol, Silber Sulfadiazin, Atropin, } \\
\text { FM }\end{array}$ & Keratekt., BHL & $\begin{array}{l}\text { Korneanarbe, } \\
\text { Visus erhalten }\end{array}$ \\
\hline 15 & Z: Pilzhyphen & - & $\begin{array}{l}\text { Amphotericin B, Silber-Sulfadiazin, } \\
\text { Atropin, FM }\end{array}$ & - & $\begin{array}{l}\text { Korneanarbe, } \\
\text { Visus erhalten }\end{array}$ \\
\hline 16 & Z: Pilzyhyphen & Asperg. sp. & Miconazol, Atropin, FM, & Keratekt., BHL & $\begin{array}{l}\text { Korneanarbe, } \\
\text { Visus erhalten }\end{array}$ \\
\hline 17 & Z: Pilzhyphen & Asperg. sp. & Fluconazol, Atropin, FM, AB & Keratekt., BHL & $\begin{array}{l}\text { Korneanarbe, } \\
\text { Visus erhalten }\end{array}$ \\
\hline 18 & Z: Pilzhyphen & - & Fluconazol, Atropin, Vedaprofen & Keratekt., BHL & $\begin{array}{l}\text { Korneanarbe, } \\
\text { Visus erhalten }\end{array}$ \\
\hline
\end{tabular}

\section{Z: Zytologie B: Biopsie BHL Bindehautlappen}

sultat verfügbar ist.

Aspergillus sp. hat die Tendenz die Hornhaut vollständig zu durchwachsen und sich in tiefen Schichten des Stromas oder sogar in der Vorderkammer festzusetzen. Die Penetration der üblichen Antimykotika ist teilweise ungenügend, so daß Pilze im Bereich der Deszemet'schen Membran oft nicht erreicht 
werden. Bei massivem Absterben von Pilzen kommt es zur Freisetzung von Endotoxinen, was die Uveitis in den ersten Tagen der Behandlung massiv verstärkt. Es ist aus diesem Grund vorteilhaft, wenn die Pilzbeladung der Kornea vorgängig durch eine lamelläre Keratektomie, oder zumindest durch ein gutes Debridement reduziert wird. Außerdem hat es sich bewährt, in den ersten Tagen die Antimykotika nur 3 bis $4 \times$ täglich anzuwenden, und erst danach die Therapiefrequenz auf 6-8 x täglich zu erhöhen. Im Anschluß an die Keratektomie, vor allem bei tiefen Läsionen, empfiehlt sich das Einnähen eines gestielten Bindehautlappens. Um eine adäquate Therapie zu gewährleisten muss in der Regel ein subpalpebrales Spülsystem verankert werden. Die medikamentelle Behandlung muss über mehrere Wochen hinweg fortgesetzt werden. Wenn möglich ist der Behandlungserfolg durch wiederholte zytologische Untersuchungen zu überprüfen.

Um die Narbenbildung so gering wie möglich zu halten, kann im Verlauf der Therapie auch ein lokaler nicht-steroidaler Entzündungshemmer, wie zum Beispiel Diclofenac-Na verabreicht werden. Auf keinen Fall sollen Kortikosteroide zum Einsatz kommen.

Die Keratomykose bleibt auch bei rechtzeitiger Diagnose und entsprechender Behandlung eine schwerwiegende Augenerkrankung beim Pferd, bei welcher es in vielen Fällen zum Verlust des Auges kommen kann.

1 Cytobrush ${ }^{\circledR}$, Medscan, Malmö, Schweden

2 Natacyn ${ }^{\circledR}$ Alcon Laboratories, Cham, Schweiz

3 Pima-Biciron ${ }^{\circledR}$ N, S\&K Pharma, Perl, Deutschland

4 Amphotericin B 50 mg Tr. Subst., Bristol-Myers Squibb, München, Deutschland

5 Dactarin ${ }^{\circledR}$, Janssen-Cilag AG, Neuss, Deutschland

6 Diflucan ${ }^{\circledR}$, Pfizer, Zug, Schweiz

7 Sempera ${ }^{\circledR}$ Liquid, Janssen-Cilag AG, Neuss, Deutschland

8 Flammazine ${ }^{\circledR}$ Solvay Pharma AG, Bern, Schweiz

9 Betaisodona ${ }^{\circledR}$ Lsg., Mundipharma, Limburg, Deutschland

10 Voltaren ${ }^{\circledR}$ Ophtha, CibaVision Ophthalmics, Hettlingen, Schweiz

11 Finadyne ${ }^{\circledR}$, Biokema SA, Crissier, Schweiz

12 Quadrisol@, Veterinaria AG, Zürich, Schweiz

13 Atropin ${ }^{\circledR}$ 1\%, 2\%, CibaVision Ophthalmics, Hettlingen, Schweiz

14 Sprenglösung nach Hollwich, Kantonsapotheke Zürich RP. Complexon

Atropin. sulfuric

Cocain. hydrochloric.

Natr. chlorat.

Sol. Adrenalin.

Desogen. so. $1 \%$

Natr. hydric. sol. $2 \mathrm{~N}$ quant. sat. auf $\mathrm{pH} 5.5$

Aqua bidest.

(Sterilisation bei $100^{\circ} \mathrm{C}$ )

15 Ocular Lavage Kit®, Cook Medical, Sursee, Schweiz

\section{Literatur}

Andrew, S., D.E.Brooks, P.J. Smith, K.N. Gelatt, N.T. Chmielewski and C.J.G. Whittaker (1998): "Equine ulcerative keratomycosis: visual outcome and ocular survival in 39 horses (1987-1996)." Equine Vet J 30, 109-116.

Ball, M., W.C. Rebhun, J.E. Gaarder and V. Patten (1997): "Evaluation of itraconazole- dimethyl sulfoxide ointment for treatment of keratomycosis in nine horses." J Am Vet Med Assoc 211, 199-203.
0.33

1.00

0.37

Barnett, K., S.M. Crispin, J.D. Lavach and A.G. Matthews (1995): Color Atlas and Text of Equine Ophthalmology. London, Mosby-Wolfe.

Baver, G., B.M. Spiess and H. Lutz, H (1996): "Exfoliative cytology of conjunctiva and cornea of domestic animals: A comparison of four collecting techniques." Vet Comp Ophthalmol 6, 181-186.

Baver, G. (1999): Exfoliative cytology of the cornea and conjunctiva of domestic animals. Department of Veterinary Surgery. Thesis, University of Zurich.

Behrens-Baumann, W. (1991): Pilzerkrannkungen des Auges. Stuttgart, Enke Verlag.

Behrens-Baumann, W. (1997): "Diagnosis and therapy of fungal keratitis - a review." Klin Monatsbl Augenheilkd 210, aA10-3.

Behrens-Baumann, W. (1997): "Topical antimycotics in ophthalmology." Ophthalmologica 211 (Suppl 1), 33-8.

Bron, A., T. Cellier, B. Delbosc, X. Piquot and J. Royer (1990): "Keratomycoses: practical aspects." Bull Soc Ophtalmol Fr 90, 869-72.

Brooks, D. (1999): Equine ophthalmology. Veterinary Ophthalmology. K. Gelatt. Philadelphia, Lippincott Williams \& Wilkins. 1.

Brooks, D. E., S.E. Andrew and M.P. Nasisse (1998): "Antimicrobial susceptibility patterns of fungi isolated from horses with ulcerative keratomycosis." Am J Vet Res 59, 138-42.

Coad, C. T., N.M. Robinson and K.R. Wilhelmus (1985): "Antifungal sensitivity testing for equine keratomycosis." Am J Vet Res 46(3): 676-8.

Davidson, M. (1991): Equine ophthalmology. Textbook of Veterinary Ophthalmology. Gelatt. KN. Philadelphia, Lea \& Febiger.

McLaughlin, S. A., A.H. Brightman, L.C. Helper, J.P. Manning and J.E. Tomes (1983): "Pathogenic bacteria and fungi associated with extraocular disease in the horse." J Am Vet Med Assoc 182, 241-2.

Negroni, R. and A. I. Arechavala (1993): "Itraconazole: pharmacokinetics and indications." Arch Med Res 24, 387-93.

O'Day, D. M. (1990): "Orally administered antifungal therapy for experimental keratomycosis." Trans Am Ophthalmol Soc 88, 685-725.

Pickett, J. (1995): Intraocular injection of miconazole for the treatment of fungal endophthalmitis in horses. 26th annual meeting of the Am Coll Vet Ophthalmol, Newport RI.

Plempel, M. (1984): "Reduction of the in vivo virulence of Candida albicans by pretreatment with subinhibitory azole concentrations in vitro." Dermatologica (169), 11-18.

Regnier A., R.D. Whitley, P. Benard and M. Bonnefoi (1986): "Effect of flunixin meglumine on the breakdown of the blood-aqueous barrier following paracentesis in the canine eye." J Ocul Pharmacol 2, 165 70 .

Samuelson DA., T.L.Andresen and R.M. Gwin (1984): "Conjunctival fungal flora in horses, cattle, dogs, and cats." J Am Vet Med Assoc 184, 1240-2.

Spiess, B. (1994): "Therapieverfahren bei Hornhautverletzung, Keratitis und Ulcus corneae des Pferdes." Prakt Tierarzt 75, 32-37.

Thakar, M. (1994): "Oral fluconazole therapy for keratomycosis." Acta Ophthalmol (Copenh) 72, 765-7.

Urbak, S. and T. Degn (1994): "Fluconazole in the management of fungal ocular infections." Ophthalmologica 208, 147-156.

Vaipayee, R. B., S. K. Gupta, U. Bareja and K. Kishore (1990): "Ocular atopy and mycotic keratitis." Ann Ophthalmol 22, 369-72.

ad $100 \mathrm{ml}$
$2.0 \mathrm{ml}$
Prof. Dr. B. M. Spiess

Veterinär-Chirurgische Klinik

Winterthurerstrasse 260

$\mathrm{CH}$-8057 Zürich

Tel. +41-7-635-8404

Fax +47-7-635-8905

Email bspiess@vetchir.unizh.ch 